\title{
Differentiation of progenitors in the liver: a matter of local choice
}

\author{
Luke Boulter, Wei-Yu Lu, and Stuart J. Forbes \\ Medical Research Council Centre for Regenerative Medicine, University of Edinburgh, Edinburgh, United Kingdom.
}

\begin{abstract}
The liver is a complex organ that requires multiple rounds of cell fate decision for development and homeostasis throughout the lifetime. During the earliest phases of organogenesis, the liver acquires a separate lineage from the pancreas and the intestine, and subsequently, the liver bud must appropriately differentiate to form metabolic hepatocytes and cholangiocytes for proper hepatic physiology. In addition, throughout life, the liver is bombarded with chemical and pathological insults, which require the activation and correct differentiation of adult progenitor cells. This Review seeks to provide an overview of the complex signaling relationships that allow these tightly regulated processes to occur.
\end{abstract}

\section{Introduction}

Progenitor cells have highly regulated mechanisms governing proliferation and differentiation to achieve accurate tissue repair. In several organ systems, the hierarchy of stem cells, transit amplifying-progenitor cells, and differentiated mature cells is well described, including the signaling pathways that define these stages (1-4), though much remains to be characterized for this hierarchy in liver. The adult liver has an impressive regenerative capability, and classical experiments in rats following partial hepatectomy demonstrated that the liver can regrow to its original size within ten days. This capability is utilized in clinical scenarios in which partial hepatectomy is used to resect liver tumors, and in living donor transplantation in which a portion of the liver is taken from a donor and transplanted into a recipient, with both remnants regrowing into a functional liver mass. This regeneration occurs through division of the mature epithelial cells (hepatocytes and cholangiocytes) within the liver, which leave their normal mitotically quiescent state, termed $\mathrm{G}_{0}$, and enter cell cycle and mitosis.

During chronic liver injury this normally efficient renewal from mature epithelial cells becomes impaired. In many liver diseases, such as chronic viral hepatitis (5) and non-alcoholic fatty liver disease (6), hepatocytes become senescent and unable to efficiently regenerate the parenchyma. In this scenario, hepatic progenitor cells (HPCs) become activated and are sufficient to regenerate the biliary and hepatocellular epithelium $(7,8)$. The biology of HPCs is less studied in comparison to analogous progenitor cells in other adult tissues, and markers that delineate the stem versus transit amplifying populations are not clearly defined (9-11).

While the putative adult liver stem cell is often thought to reside within the canals of Hering, located at the terminal branches of the biliary tree, multiple lines of recent work suggest that a label-retaining stem cell might exist throughout the biliary network $(12,13)$ and even in the common bile duct $(14,15)$. One model posits that these putative stem cells may be differentially activated in diverse disease patterns and may have different differentiation potencies depending on their location, though this idea has not yet been formally addressed. What is known is that this putative stem cell gives rise to a transit-amplifying population of

Conflict of interest: The authors have declared that no conflict of interest exists. Citation for this article: J Clin Invest. 2013;123(5):1867-1873. doi:10.1172/JCI66026.
HPCs. Mechanisms of HPCs have been increasingly studied, and a broad class of factors that can activate the proliferation of HPCs has been identified $(16,17)$. To restore function to the damaged liver, these bipotential HPCs must differentiate into hepatocytes, the key metabolic cells of the liver, and cholangiocytes, which line the biliary tree and transport bile into the intestine. The mechanism by which HPCs differentiate into these two key epithelial types is poorly defined, but recent advances in transgenic mouse technology and the ability to manipulate HPCs in vitro and in vivo has shown that the fate of HPCs depends not only upon which signaling cascades are activated within the HPCs, but also the disease context in which HPCs evolve.

\section{Expansion of HPCs is a multifactorial process}

HPCs are facultative and not activated in healthy liver. The induction of HPC proliferation is critical to generate sufficient numbers of transit-amplifying cells, which can subsequently differentiate into the mature hepatic epithelia. Multiple signaling pathways have been identified as mitogenic for HPCs (for a comprehensive review, see ref. 18). TGF- $\beta$ is a potent mitogen associated with the inflammatory response that is able to act differentially on TGF$\beta R 2$ expressed by hepatocytes and HPCs through variable glycosylation of the TGF- $\beta$ receptor (19). Hepatocyte proliferation is restricted by TGF- $\beta$, although HPCs with glycosylated TGF- $\beta$ R are not repressed by TGF- $\beta$ and can proliferate in an inflammatory environment. TNF-like weak inhibitor of apoptosis (TWEAK) acts though its receptor, Fn14, activating NF- $\kappa$ B signaling and potently inducing HPC proliferation. Knockout of either the TWEAK ligand or the Fn14 receptor results in a severely abrogated HPC response; moreover, exogenous injection of TWEAK is sufficient to induce HPCs in an undamaged mouse liver $(16,17)$.

\section{In vitro assessment of the stemness and differentiation capacity of HPCs}

Due to limitations in the available lineage-tracking system in the adult liver, much of our understanding of HPC differentiation has largely been defined in vitro. HPCs are isolated, and their ability to form clones (clonogenicity) and to differentiate into multiple lineages (multipotentiality) can be assayed.

Wang et al. sought to identify a bipotential population of cells with self-renewing capacity that were transplantable and could contribute to hepatic parenchyma in vivo (20). c-kit-positive 
A

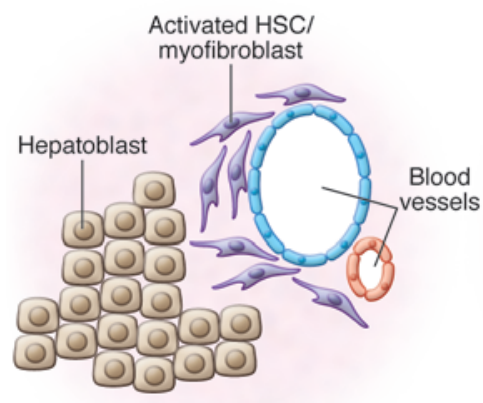

Embryonic day 14.5
B

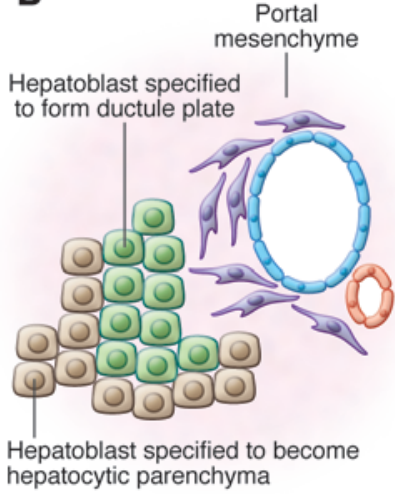

Embryonic day 16.5
C

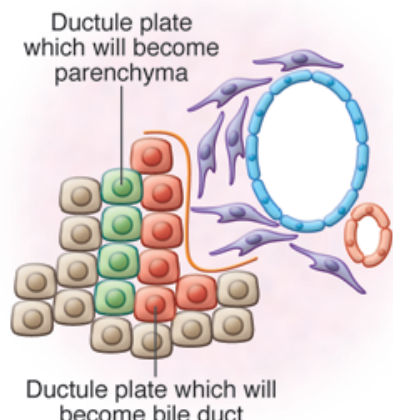

become bile duct

Embryonic day 17.5
D Mature bile duct
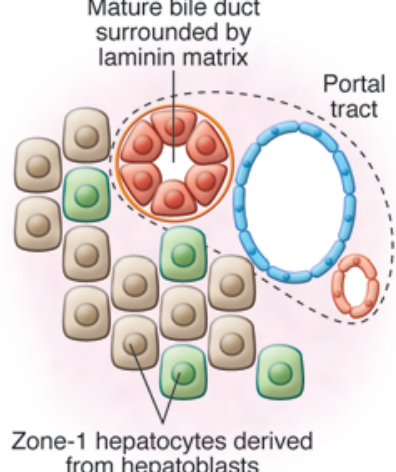

from hepatoblasts

Mature adult

\section{Figure 1}

Liver development involves multiple phases of specification and differentiation. (A) During liver development, the liver bud is specified from the remainder of the endoderm by Wnt expression from the cardiac mesoderm. HSC, hepatic stellate cell. (B) Once specified, the endoderm matures into fetal hepatoblasts that are capable of bilineage differentiation into hepatocytes and cholangiocytes. (C) Spatial heterogeneity across the developing liver results in the specification of the ductal plate though activation of the Notch signaling pathway, in close proximity to what will ultimately become the portal tract. (D) Once specified, the ductal plate is patterned, and the side adjacent to the portal mesenchyme closely associates with laminin and expresses high levels of Sox9, whereas the side adjacent to the developing parenchyma expresses TGF- $\beta-$ mediated C/EBP and contributes to zone-1 hepatocytes.

progenitor cells were isolated from wild-type donors and transplanted into fumaryl acetoacetate hydrolase-null $\left(\mathrm{Fah}^{-/}\right)$recipient mice, in which hepatocytes lack a tyrosine metabolic pathway (20). The c-kit-positive cells differentiated into genetically normal hepatocytes, which proliferated to rescue the $\mathrm{Fab}^{-/}$phenotype (20). In later studies this group generated a suite of antibodies against HPCs, such as MIC1-1C3 and OC-2, which were used to identify a population of putative HPCs (21). However, a number of the antibodies generated in these studies also cross-react with bile ducts in the healthy adult mouse, and as such these markers may not isolate a pure HPC population. This problem was addressed by culturing sorted cell populations in vitro at clonal density using selection based on the expression pattern $\mathrm{CD}^{-} 5^{-} \mathrm{CD} 11 \mathrm{~b}^{-} \mathrm{CD} 31^{-} \mathrm{MIC} 1-1 \mathrm{C}^{+} \mathrm{C}-$ $\mathrm{D} 133^{+} \mathrm{CD} 26$; these cells were bipotent, with the capacity to differentiate into two distinct cell types, hepatocytes and cholangiocytes (22). Furthermore, additional data suggested that a liver-regenerating cell resides within the epithelial cell adhesion molecule-positive (EPCAM-positive) population within the uninjured adult liver, and that these cells are capable of forming epithelial colonies with bilineage potential (23). However, the identity of these cells within the EPCAM-positive pool remains enigmatic.

\section{Lineage tracing in the adult liver}

The contribution of progenitors to tissue turnover and regeneration is difficult to address in adult organs, which are already structured and contain terminally differentiated cells. To this end, several stem cell lineage-tracing tools have been developed to assess the location of HPCs and the mechanisms governing their contribution to regeneration. The basic principle of lineage-tracing animals is that the putative stem or progenitor cell is indelibly marked; as such, any progeny arising from this cell will also carry the same distinct mark. The most common method of lineage tracing is to use an inducible, lineage-specific Cre-recombinase crossed with a mouse that harbors a flox-stop-flox reporter (such as GFP or LacZ). Following induction, Cre recombinase excises the stop codon and the stem cell is subsequently labeled irreversibly with either GFP or LacZ. Therefore, its progeny can be tracked during tissue homeostasis and regeneration (24).

The initial liver studies from Linda Greenbaum and colleagues identified Foxl1 as a marker of adult HPCs (25). This was an important advance because previous attempts to track cells in the adult liver had been limited primarily to markers of adult HPCs, including the cytokeratins Krt7 and Krt19 and other liver-restricted markers (e.g., $\alpha$-fetoprotein) expressed on fetal hepatoblasts, and, as such, made adult analysis of these tissues impossible $(26,27)$. The use of Foxl1Cre flox-stop-flox LacZ reporter mice demonstrated that HPCs could contribute to mature hepatocytes when livers were damaged and progenitor cells were activated, but the number of positive hepatocytes was rare $(0.5 \%$ Foxl 1 and hepatic nuclear factor $4 \alpha[\mathrm{HNF} 4 \alpha]$ dual-positive hepatocytes) and likely too low for mechanistic studies.

The use of inducible Cre provides an attractive alternative to constitutive reporter systems. In this context the Cre recombinase is fused to an estrogen response element, which upon administration of 4-hydroxytamoxifen results in the translocation of the fusion protein to the nucleus, where the enzyme can then induce recombination (28). Administration of an inducing agent means labeling of HPCs can be strictly timed, and the cellular progeny that result from this recombination can be tracked over time. Utilizing this inducible Cre technology under the control of the Sox 9 transcriptional control elements, Furuyama et al. described how Sox9 is expressed in HPCs and biliary epithelium in the regenerating liver (29). Using both an inducible Sox9-Cre/LacZ mouse and also a Sox9-GFP reporter mouse in which GFP is expressed in Sox9expressing cells, the authors found Sox $9^{+}$cells in close proximity to the biliary tree in normal liver. Interestingly, when healthy animals were left for up to 12 months, the parenchyma of these animals was replaced by cells of a Sox 9 origin, the putative HPCs (29). This result was remarkable and challenged the dogma about normal homeostatic mechanisms in the liver - rather than the mature 


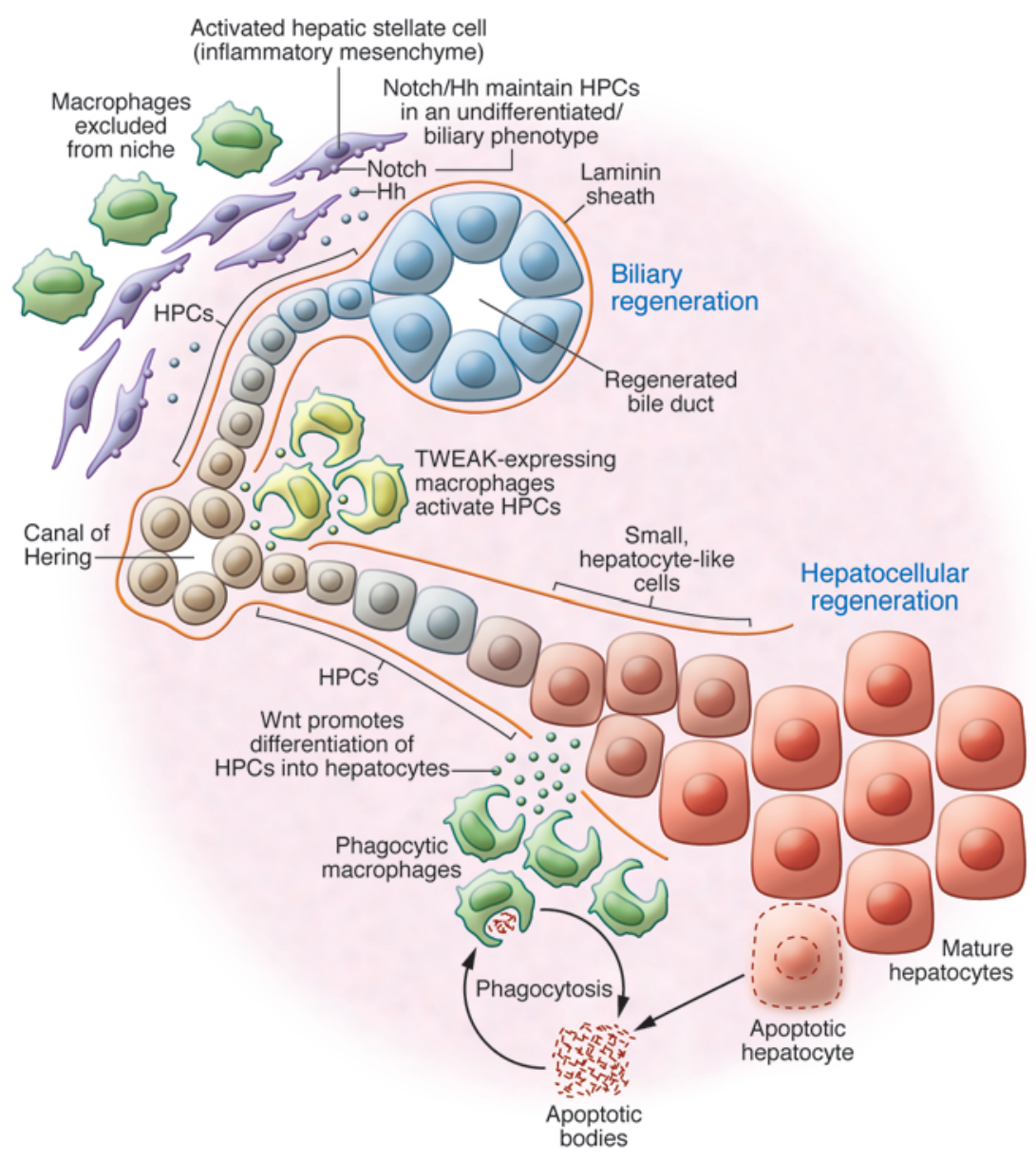

\section{Figure 2}

HPCs are involved in a complex microenvironment and are subject to multiple signals. Regeneration in the liver can be broadly characterized into hepatocellular or biliary regeneration. In the former context, progenitor cells irradiate from the portal tracts, in which they are sheathed in laminin, which facilitates their expansion. Upon exit from the laminin niche, these cells are subject to differentiation cues, such as Wnt and HGF, which activate the pro-hepatocyte transcriptional cascade in HPCs. In biliary regeneration, HPCs emerge in a similar fashion, but they remain in the laminin ECM, in which fibroblasts are able to influence their maturation though activation of the Notch signaling pathway. This pathway influences the activation of the HNF6/HNF1 $\beta$ transcriptional network to correctly specify cholangiocytes. parenchyma being responsible for maintaining itself, turnover of the healthy liver was progenitor mediated. Furthermore, when Sox9-Cre/LacZ mice were injured there was a startling expansion of Sox9-positive progeny that quickly restored normal epithelial function (29). However, in 2011 a second paper investigating the embryonic origin of HPCs shed some light on these exceptional Sox9 results (30).

Work from Frédéric Lemaigre's group clearly demonstrated that the Sox9-positive bile duct and progenitor cell are deposited from the ductal plate during hepatic ontogeny; in a thorough study the authors demonstrated that the embryonic ductal plate gives rise not only to biliary epithelium, as previously expected, but also to HPCs and to a subpopulation of hepatocytes in periportal zone 1 of the adult liver (30). Moreover, upon tamoxifen administration to adult Sox9 reporter mice, the zone 1 hepatocytes upregulated Sox9. This control suggests that the Sox9-positive cells identified by Furuyama et al. (29) may in fact be periportal hepatocytes that upregulate Sox 9 and activate the transgene in response to tamoxifen administration.

The work from Uemoto and Furuyama (29), however, is not without considerable merit because this paper clearly demonstrates a streaming gradient of cells that arise at the portal tract and then divide and potentially migrate through the zones of the liver until they reach the central vein. This confirms the so-called "streaming liver hypothesis" originally described by Zajicek and colleagues $(31,32)$.
The culmination of these lineage-tracing strategies has resulted in an important recently published work by the Leclercq group (7). Using osteopontin-1 as a marker, the authors demonstrate that HPCs express osteopontin (a glycoprotein that marks HPCs), emerge from bile duct, and are capable of directly differentiating into hepatocytes (7). Importantly, HPCs regenerated hepatocytes following chronic hepatocyte injury $(2.4 \%$ of hepatocytes were positive for GFP after two weeks of a choline-deficient, ethioninesupplemented $[\mathrm{CDE}]$ diet), but not following biliary injury and regeneration (3,5-diethoxycarbonyl-1,4-dihydrocollidine diet, with which $0 \%$ YFP-positive hepatocytes were seen), demonstrating that the HPC microenvironment is critical for HPC expansion and differentiation, as we have previously suggested (33). Interestingly, the HPCs radiated from their niche to the site of injury but contributed little to hepatocyte regeneration during the injury on the $\mathrm{CDE}$ diet. However, during recovery with a normal diet, HPCs differentiated into mature, replication-competent hepatocytes that could then maintain parenchymal regeneration themselves. This insight has relevance to the study of human liver disease, which may have intermittent relapsing-remitting disease activity.

There has been debate in the liver field as to whether hepatic stellate cells are capable of undergoing mesenchymal-epithelial transition (MET), thereby contributing to parenchymal regeneration. Work from Yang and colleagues utilized a constitutive GFAP-Cre line, which labeled the biliary tree and hepatic stellate cells (34). This study demonstrated for the first time that GFAP-expressing 
cells could be traced into hepatocytes, and by analyzing the kinetics of disease recovery, Yang et al. concluded that the parenchyma can be regenerated from non-epithelial, $\mathrm{GFAP}^{+}$cells (34). However, the use of a constitutive GFAP-Cre expression system does not fully exclude the possibility that GFP-labeled hepatocytes are derived from GFAP-expressing bile ducts. This observation, however, offers a tantalizing suggestion that stellate cells may have a progenitor role in the adult liver; this has been investigated by other groups in the liver $(35,36)$ and other tissues (37). Of course stellate cells are also subject to regional signaling in the liver, and multiple developmental regulators, including Necdin-Wnt (38-40) and Hedgehog, have been implicated in the differentiation of hepatic stellate cells into fibroblasts, the latter of which control cellular metabolism to regulate fate (41). Intriguingly, differentiation of these fibroblasts is in part mediated through epigenetic regulation of PPAR $\gamma$ (42-44), although there is little evidence to indicate which signals might facilitate MET.

Do these murine studies shed any light on the human condition? Alison and colleagues have harnessed the inability of the cell to repair mutations in mitochondrial DNA to ask whether human livers have such progenitors $(8,45)$. By identifying cells in which the mitochondria have a COX mutation, this group described patches of epithelium in the liver in which the mitochondria were mutated, and when random, discrete regions of the patch were analyzed the mitochondrial mutation was consistent across the sample. This result suggests that the COX mutation carried in these mitochondria arose from a common ancestor of cells throughout the patch. Of course, this does not necessarily point to a progenitor cell, but if both hepatocytes and cholangiocytes could be identified with the same mitochondrial mutation, then this would be compelling evidence that in the adult human liver both key epithelial types can originate from a common precursor.

\section{Inspiration from development: liver patterning during ontogeny}

During development, embryonic progenitors (hepatoblasts) must make lineage decisions similar to adult HPCs and form the biliary tree and hepatocytes. Once the liver bud has formed from the primitive endoderm, epithelial lineage separation occurs; a population of hepatoblasts adjacent to the portal mesenchyme is specified and forms the ductal plate (46). This process requires the differentiation of hepatoblasts into the bile ducts and repression of the ductal phenotype in the developing hepatocytes (47-49), summarized in Figure 1. The delineation of bile duct from the rest of the parenchyma occurs early in development and is initiated by asymmetry of the ductal plate where, in E-cadherin-positive cells, HNF4 $\alpha$ and Sox9 are asymmetrically expressed on the parenchymal and the portal faces, respectively. This asymmetry of the ductal plate is enhanced through deposition of laminin on the portal side of the presumptive bile duct, whereas the repression of biliary phenotype in the parenchymal side of the ductal plate is driven through TGF- $\beta$ signaling, putatively through the TGF- $\beta$ R2 (47). This regulatory asymmetry is critical, though not for the propagation of a biliary phenotype. As in Sox9 knockout livers, there is little change in the expression of HNF1 $\beta$, HNF6, and hematopoietically expressed homeobox (HEX), but rather there is a failure of hepatocyte fate repression. Without Sox9 repression of $\mathrm{C} / \mathrm{EBP} \alpha$, there is a failure to resolve the ductal plate during embryogenesis.
A critical parameter for lineage specification of the adult biliary system is the Notch signaling pathway (50). During development, this signaling cascade is critically implicated in the formation of cholangiocytes and also in the maturation and terminal patterning of the biliary tree in a multitude of species (51-53). In the normally developing liver, Notch2 is activated in the presumptive cholangiocytes by jagged 1 , which is expressed by the portal mesenchyme (54). In this context, an HNF6/HNF1 $\beta$ signaling cascade is activated as a direct result of Notch intracellular domain interactions with the promotor of $\operatorname{HNF} 6(55,56)$. Loss of Notch signaling in biliary development in mice, through genetic ablation of jagged 1 or haplosufficiency of Notch2, results in a reduction in biliary development and failure to pattern the biliary tree (57-60). The human congenital disease Alagille syndrome, which is caused by mutations in Notch pathway components, is characterized by a biliary paucity with failure to correctly resolve the ductal plate during development; this phenotype can also be observed in adult mice that harbor a fringe mutant in which Notch is inappropriately activated (61). Huppert and colleagues demonstrated that the Notch 1 intracellular domain must act in a dose-dependent fashion, showing that overexpression of the Notch 1 intracellular region during development results in a hyper-arborized biliary network, presumably at the cost of mature parenchyma (62).

Whereas the role for Notch in development is highly stereotyped, and its effect is common between species, the role for Wnt/ $\beta$-catenin signaling in the developing liver is confounded and complex. Wnt plays critical roles not only in expansion of the liver bud (63-65), but also in formation of the definitive hepatoblasts (66), biliary proliferation (67), and hepatocyte maturation $(68,69)$. As such, elucidating the temporal and spatial action of the canonical Wnt pathway is complex.

Initial transgenic studies that looked at activating the Wnt/ $\beta$-catenin signaling pathway through embryonic loss of adenomatosis polyposis coli (APC), a critical regulator of $\beta$-catenin stability, demonstrated that this signaling cascade was required for the expansion of fetal hepatoblasts (66). Furthermore, the presence of constitutively activated $\beta$-catenin resulted in hypotrophy of the developing liver with perturbed hepatoblast differentiation occurring at embryonic day E11.5 and resulted in late lethality in utero at E16.5-E18.5 (66). These data, however, are in contrast to data from the Monga group (70). Tan et al. demonstrated that embryonic deletion of the gene encoding $\beta$-catenin under the control of the FoxA3-Cre transgene (expressed by E9.5) results in a failure of liver development through reduced hepatocyte differentiation, increased hepatoblast apoptosis, and reduced hepatoblast proliferation (70). The phenotype in the embryos is only realized at E12, a timing that is similar to APC mutant embryos. These data together indicate not only that Wnt signaling is critical for fetal hepatogenesis, but also that in normal development, modulation of this pathway must be exquisitely timed for correct liver development. Interestingly, in the postnatal liver, activation of the canonical Wnt signaling pathway is required for the expansion of hepatocytes and is responsible for expansion of the liver. Selective postnatal loss of $\beta$-catenin results in a reduced organ size, and as such suggests a conserved functional outcome of these signaling pathways during development and maturation (71). Intriguingly, targets of $\beta$-catenin such as lymphoid enhancer binding factor 1 and $\mathrm{Myc}$ are directly responsible (in association with liver-enriched transcription factors) for patterning the developing liver and are critical for correct metabolic zonation (68). 


\section{Mechanisms of differentiation during adult regeneration}

Whereas the mechanisms of lineage specification during hepatic ontogeny are increasingly understood, the cues by which HPCs in the adult liver are specified into different lineages are not well described in situ. However a handful of studies have looked at the mechanism by which HPCs differentiate in vivo. The HGF receptor c-Met is required for the maturation of HPCs into hepatocytes, and until recently this was all that was known about the potential mechanisms by which HPCs become hepatocytes (72). More recently, lineage-tracing evidence has emerged demonstrating that inhibition of laminin signaling with iloprost, a synthetic analog of prostacyclin $\mathrm{PGI}_{2}$, in HPCs during regeneration results in the precocious differentiation of HPCs into hepatocytes (7), reiterating previous descriptive work examining laminin in the HPC response $(73,74)$.

We have identified a re-establishment of the embryonic state in regeneration; Notch and Wnt are required for HPC differentiation, and their interaction is necessary for appropriate delineation of hepatocellular versus biliary fate (33). During activation of HPCs in biliary diseases of both mouse and human, HPCs express the receptors Notch1 or Notch2, respectively; these are activated though interaction with jagged 1 . In the mouse, the periportal fibroblasts that surround the regenerating bile ducts express this ligand and appear to recapitulate in some regards the portal mesenchyme seen in development $(33,75)$.

This Notch-high state appears to delineate the default pathway for the development of HPCs into cholangiocytes, and critically this pathway is inhibited during regeneration of hepatocytes through the ubiquitin ligase Numb. In HPCs undergoing hepatocyte differentiation, Numb transcription is activated by $\beta$-catenin and as such represents a critical node at which the Notch and Wnt pathways can interact to enable lineage specification. In recent years, macrophages have been described as a source of Wnt, often in cancer, though some regeneration studies have also described macrophage-derived Wnt $(76,77)$. Ablation of macrophages during hepatocyte regeneration removed the stimulus for HPCs to become hepatocytes; instead, they differentiated into cholangiocytes and formed biliary structures. These results have also been confirmed in independent studies in which macrophages have been depleted in the liver (78). Notably, phagocytosis of the hepatocyte debris promoted profound Wnt upregulation in macrophages, providing a critical link between hepatocyte death and HPC fate that enables co-ordinated and appropriate tissue renewal. Furthermore, this model provides a mechanism linking local tissue injury and inflammation patterns of specific diseases with the extra- and intracellular signaling controls that govern HPC fate.

Clinically, in the context of severe liver injury accompanied by a failure of hepatocyte regeneration and consequent functional decrease in parenchymal mass, a marked ductal reaction occurs, which likely represents a regenerative response that is "too biliary" $(79,80)$. In such situations, promoting hepatocytic differentiation of progenitors may provide a legitimate future target for drugs and small molecules. Indeed, by genetically expressing stabilized Wnt in mouse liver progenitors, we have found that HPCs can be coaxed in vivo into a hepatocyte fate rather than a biliary fate (33).

\section{Parallels in cancer}

Recent intriguing work in mice suggests that the overactivation of the Notch signaling pathway (through ectopic expression of the Notch 1 intracellular domain in hepatocytes in vivo) can transdifferentiate these mature cells into cancerous cholangiocytes $(81,82)$. This phenomenon obviously parallels the mechanisms of biliary development discussed earlier, in that repression of the hepatocyte phenotype and promotion of the biliary phenotype can specify cells toward a cholangiocyte phenotype. In these mouse models of cancer, Notch deregulation clearly results in the propagation of a naive phenotype that is carcinogenic. Similarly, Wnt deregulation has been implicated in the development of hepatocellular carcinoma (83). These cancers occur most commonly in the context of chronic liver injury and regeneration, and emerging evidence suggests that parallels in the signaling pathways linking development, regeneration, and cancer may enable new treatment modalities to promote healthy liver regeneration and inhibit the formation of liver cancer.

\section{Conclusions and perspectives}

Clearly the activation and evolution of HPCs during disease is one of multiple pathogenic phases analogous to disease onset in other adult tissues. In this model a resident (and yet to be identified) hepatic stem cell is activated, giving rise to a transit-amplifying pool known as HPCs. These cells then undergo rapid division and expansion, likely driven by inflammatory cues, principally TWEAK. These HPCs evolve in a laminin-rich microenvironment, which is necessary to facilitate their expansion and maintain their naivete. In this steady state, once the niche is established, HPCs are subject to multiple classical signaling cascades that are context (disease) dependent. If the HPCs are exposed to Notch signaling whilst in the niche, they form cholangiocytes and maintain a laminin rich basement membrane around themselves. When HPCs exit the laminin sheath, these cells are exposed to multiple ligands from their local environment, such as macrophage derived Wnt or HGF, which acts through c-Met expressed by HPCs and initiates the activation of a pro-hepatocyte signaling cascade and results in differentiation of HPCs into hepatocytes (Figure 2).

Regeneration in the liver is undoubtedly an important clinical target. The ability to influence HPCs to proliferate and to regulate their differentiation may ultimately lead to exciting new therapies for chronic liver diseases. Until recently, HPC research has moved slowly compared with research for other organ systems such as gut, blood, and skin. Difficulties identifying HPCs in vivo, limited tools available to identify their targets, and difficulties culturing highly pure populations capable of modeling the in vivo situation have been at least partly overcome, making the present a prime time for progress in this field.

\section{Acknowledgments}

The authors thank the Cancer Research UK (CRUK), Wellcome Trust, Medical Research Council (MRC), and Sir Jules Thorn Trust for funding. The authors also thank Robert Walker for his comments on the manuscript. Financial support was received from the Sir Jules Thorn Trust, MRC, CRUK, Wellcome Trust, and Scottish Enterprise.

Address correspondence to: Stuart J. Forbes, MRC Centre for Regenerative Medicine, SCRM Building, University of Edinburgh, Edinburgh BioQuarter, 5 Little France Drive, Edinburgh, EH16 4UU. Phone: 44.131.651.9515; Fax: 44.131.651.9501; E-mail: stuart.forbes@ed.ac.uk. 
1. Barker $\mathrm{N}$, et al. Identification of stem cells in small intestine and colon by marker gene Lgr5. Nature. 2007;449(7165):1003-1007.

2. Watt FM, Jensen KB. Epidermal stem cell diversity and quiescence. EMBO Mol Med. 2009;1(5):260-267.

3. Jensen KB, et al. Lrig1 expression defines a distinct multipotent stem cell population in mammalian epidermis. Cell Stem Cell. 2009;4(5):427-439.

4. Rawlins EL, et al. The role of Scgb1a1+ Clara cells in the long-term maintenance and repair of lung airway, but not alveolar, epithelium. Cell Stem Cell. 2009;4(6):525-534.

5. Marshall A, et al. Relation between hepatocyte G1 arrest, impaired hepatic regeneration, and fibrosis in chronic hepatitis $\mathrm{C}$ virus infection. Gastroenterology. 2005;128(1):33-42.

6. Yang $\mathrm{S}$, et al. Oval cells compensate for damage and replicative senescence of mature hepatocytes in mice with fatty liver disease. Hepatology. 2004;39(2):403-411.

7. Espanol-Suner R, et al. Liver progenitor cells yield functional hepatocytes in response to chronic liver injury in mice. Gastroenterology. 2012; 143(6):1564-1575

8. Fellous TG, et al. Locating the stem cell niche and tracing hepatocyte lineages in human liver. Hepatology. 2009; 49(5):1655-1663.

9. Schotanus BA, van den Ingh TS, Penning LC, Rothuizen J, Roskams TA, Spee B. Cross-species immunohistochemical investigation of the activation of the liver progenitor cell niche in different types of liver disease. Liver Int. 2009;29(8):1241-1252.

10. Watt FM, Lo CC, Silva-Vargas V. Epidermal stem cells: an update. Curr Opin Genet Dev. 2006;16(5):518-524.

11. Watt FM. Epidermal stem cells: markers, patterning and the control of stem cell fate. Philos Trans $R$ Soc Lond B Biol Sci. 1998;353(1370):831-837.

12. Sell S. The hepatocyte: heterogeneity and plasticity of liver cells. Int J Biochem Cell Biol. 2003;35(3):267-271.

13. Sell S. Comparison of liver progenitor cells in human atypical ductular reactions with those seen in experimental models of liver injury. Hepatology. 1998;27(2):317-331.

14. Cardinale V, et al. Multipotent stem/progenitor cells in human biliary tree give rise to hepatocytes, cholangiocytes, and pancreatic islets. Hepatology. 2011;54(6):2159-2172

15. Carpino G, et al. Biliary tree stem/progenitor cells in glands of extrahepatic and intraheptic bile ducts: an anatomical in situ study yielding evidence of maturational lineages. J Anat. 2012;220(2):186-199.

16. Tirnitz-Parker JE, et al. Tumor necrosis factor-like weak inducer of apoptosis is a mitogen for liver progenitor cells. Hepatology. 2010;52(1):291-302.

17. Jakubowski A, et al. TWEAK induces liver progenitor cell proliferation. JClin Invest. 2005;115(9):2330-2340.

18. Bird TG, Lorenzini S, Forbes SJ. Activation of stem cells in hepatic diseases. Cell Tissue Res. 2008;331(1):283-300.

19. Nguyen LN, et al. Transforming growth factor-beta differentially regulates oval cell and hepatocyte proliferation. Hepatology. 2007;45(1):31-41.

20. Wang X, Foster M, Al-Dhalimy M, Lagasse E, Finegold M, Grompe M. The origin and liver repopulat ing capacity of murine oval cells. Proc Natl Acad Sci US A. 2003;100(suppl 1):11881-11888.

21. Dorrell C, et al. Surface markers for the murine oval cell response. Hepatology. 2008;48(4):1282-1291.

22. Dorrell C, et al. Prospective isolation of a bipotential clonogenic liver progenitor cell in adult mice. Genes Dev. 2011;25(11):1193-1203.

23. Okabe M, et al. Potential hepatic stem cells reside in EpCAM+ cells of normal and injured mouse liver. Development. 2009;136(11):1951-1960.

24. Kretzschmar K, Watt FM. Lineage tracing. Cell. 2012;148(1-2):33-45.

25. Sackett SD, et al. Foxl1 is a marker of bipoten- tial hepatic progenitor cells in mice. Hepatology. 2009;49(3):920-929.

26. Harada $\mathrm{N}$, et al. Intestinal polyposis in mice with a dominant stable mutation of the beta-catenin gene. EMBO J. 1999;18(21):5931-5942.

27. Kellendonk C, Opherk C, Anlag K, Schutz G, Tronche F. Hepatocyte-specific expression of Cre recombinase. Genesis. 2000;26(2):151-153.

28. Dor Y, Brown J, Martinez OI, Melton DA. Adult pancreatic beta-cells are formed by self-duplication rather than stem-cell differentiation. Nature. 2004;429(6987):41-46.

29. Furuyama K, et al. Continuous cell supply from a Sox9-expressing progenitor zone in adult liver, exocrine pancreas and intestine. Nat Genet. 2011;43(1):34-41.

30. Carpentier R, et al. Embryonic ductal plate cells give rise to cholangiocytes, periportal hepatocytes, and adult liver progenitor cells. Gastroenterology. 2011;141(4):1432-1438

31. Arber N, Zajicek G, Ariel I. The streaming liver. II. Hepatocyte life history. Liver. 1988;8(2):80-87.

32. Zajicek G, Oren R, Weinreb $M$. The streaming liver. Liver. 1985;5(6):293-300.

33. Boulter L, et al. Macrophage-derived Wnt opposes Notch signaling to specify hepatic progenitor cell fate in chronic liver disease. Nat Med. 2012;18(4):572-579.

34. Yang L, et al. Fate-mapping evidence that hepatic stellate cells are epithelial progenitors in adult mouse livers. Stem Cells. 2008;26(8):2104-2113.

35. Kordes C, Sawitza I, Haussinger D. Hepatic and pancreatic stellate cells in focus. Biol Chem. 2009;390(10):1003-1012.

36. Kordes C, et al. CD133+ hepatic stellate cells are progenitor cells. Biochem Biophys Res Commun. 2007;352(2):410-417.

37. Chaffer CL, et al. Normal and neoplastic nonstem cells can spontaneously convert to a stem-like state. Proc Natl Acad Sci U S A. 2011;108(19):7950-7955.

38. Zhu NL, Wang J, Tsukamoto H. The Necdin-Wnt pathway causes epigenetic peroxisome proliferator-activated receptor gamma repression in hepatic stellate cells. J Biol Chem. 2010;285(40):30463-30471.

39. Jiang F, Parsons CJ, Stefanovic B. Gene expression profile of quiescent and activated rat hepatic stellate cells implicates Wnt signaling pathway in activation. J Hepatol. 2006;45(3):401-409.

40. Xiong WJ, et al. Wnt5a participates in hepatic stellate cell activation observed by gene expression profile and functional assays. World J Gastroenterol. 2012;18(15):1745-1752.

41. Chen Y, et al. Hedgehog controls hepatic stellate cell fate by regulating metabolism. Gastroenterology. 2012;143(5):1319-1329.

42. Handeli S, Simon JA. A small-molecule inhibitor of Tcf/beta-catenin signaling down-regulates PPARgamma and PPARdelta activities. Mol Cancer Ther. 2008;7(3):521-529.

43. Tsukamoto H, Zhu NL, Asahina K, Mann DA, Mann J. Epigenetic cell fate regulation of hepatic stellate cells. Hepatol Res. 2011;41(7):675-682.

44. Zeybel M, et al. Multigenerational epigenetic adaptation of the hepatic wound-healing response. Nat Med. 2012;18(9):1369-1377.

45. Fellous TG, et al. A methodological approach to tracing cell lineage in human epithelial tissues. Stem Cells. 2009;27(6):1410-1420.

46. Zaret KS, Grompe M. Generation and regeneration of cells of the liver and pancreas. Science. 2008;322(5907):1490-1494.

47. Antoniou A, et al. Intrahepatic bile ducts develop according to a new mode of tubulogenesis regulated by the transcription factor SOX9. Gastroenterology. 2009;136(7):2325-2333.

48. Beaudry JB, et al. Threshold levels of hepatocyte nuclear factor 6 (HNF-6) acting in synergy with HNF-4 and PGC-1alpha are required for time-spe- cific gene expression during liver development. Mol Cell Biol. 2006;26(16):6037-6046.

49. Laudadio I, et al. A feedback loop between the liver-enriched transcription factor network and miR-122 controls hepatocyte differentiation. Gastroenterology. 2012;142(1):119-129.

50. Bray SJ. Notch signalling: a simple pathway becomes complex. Nat Rev Mol Cell Biol. 2006;7(9):678-689.

51. Ader T, Norel R, Levoci L, Rogler LE. Transcriptional profiling implicates TGFbeta/BMP and Notch signaling pathways in ductular differentiation of fetal murine hepatoblasts. Mech Dev. 2006;123(2):177-194.

52. Kodama Y, Hijikata M, Kageyama R, Shimotohno $\mathrm{K}$, Chiba $\mathrm{T}$. The role of notch signaling in the development of intrahepatic bile ducts. Gastroenterology. 2004;127(6):1775-1786

53. Lorent K, Moore JC, Siekmann AF, Lawson N, Pack M. Reiterative use of the notch signal during zebrafish intrahepatic biliary development. Dev Dyn. 2010;239(3):855-864.

54. Hofmann JJ, Zovein AC, Koh H, Radtke F, Weinmaster G, Iruela-Arispe ML. Jagged 1 in the portal vein mesenchyme regulates intrahepatic bile duct development: insights into Alagille syndrome. Development. 2010;137(23):4061-4072.

55. Vanderpool C, Sparks EE, Huppert KA, Gannon $M$, Means AL, Huppert SS. Genetic interactions between hepatocyte nuclear factor- 6 and Notch signaling regulate mouse intrahepatic bile duct development in vivo. Hepatology. 2012;55(1):233-243.

56. Tanimizu N, Miyajima A. Notch signaling controls hepatoblast differentiation by altering the expression of liver-enriched transcription factors. J Cell Sci. 2004;117(pt 15):3165-3174.

57. Kohler C, Bell AW, Bowen WC, Monga SP, Fleig W, Michalopoulos GK. Expression of Notch-1 and its ligand Jagged-1 in rat liver during liver regeneration. Hepatology. 2004;39(4):1056-1065.

58. Loomes KM, et al. Bile duct proliferation in liver-specific Jag1 conditional knockout mice: effects of gene dosage. Hepatology. 2007;45(2):323-330.

59. Lozier J, McCright B, Gridley T. Notch signaling regulates bile duct morphogenesis in mice. PLoS One. 2008;3(3):e1851.

60. McCright B, Lozier J, Gridley T. A mouse model of Alagille syndrome: Notch2 as a genetic modifier of Jag1 haploinsufficiency. Development. 2002;129(4):1075-1082.

61. Ryan MJ, et al. Bile duct proliferation in Jag1 fringe heterozygous mice identifies candidate modifiers of the alagille syndrome hepatic phenotype. Hepatology M. 2008;48(6):1989-1997.

62. Sparks EE, Huppert KA, Brown MA, Washington MK, Huppert SS. Notch signaling regulates formation of the 3-dimensional architecture of intrahepatic bile ducts in mice. Hepatology. 2010;51(4):1391-1400.

63. Goessling W, et al. APC mutant zebrafish uncover a changing temporal requirement for wnt signaling in liver development. Dev Biol. 2008;320(1):161-174.

64. Micsenyi A, Tan X, Sneddon T, Luo JH, Michalopoulos GK, Monga SP. Beta-catenin is temporally regulated during normal liver development. Gastroenterology. 2004;126(4):1134-1146

65. Ober EA, Verkade H, Field HA, Stainier DY. Mesodermal Wnt2b signalling positively regulates liver specification. Nature. 2006;442(7103):688-691.

66. Decaens T, et al. Stabilization of beta-catenin affects mouse embryonic liver growth and hepatoblast fate. Hepatology. 2008;47(1):247-258.

67. Monga SP, Monga HK, Tan X, Mule K, Pediaditakis P, Michalopoulos GK. Beta-catenin antisense studies in embryonic liver cultures: role in proliferation, apoptosis, and lineage specification. Gastroenterology. 2003;124(1):202-216.

68. Burke ZD, Reed KR, Phesse TJ, Sansom OJ, Clarke $\mathrm{AR}$, Tosh D. Liver zonation occurs through a beta- 
catenin-dependent, c-Myc-independent mechanism. Gastroenterology. 2009;136(7):2316-2324.

69. Reed KR, et al. B-catenin deficiency, but not Myc deletion, suppresses the immediate phenotypes of APC loss in the liver. Proc Natl Acad Sci U S A. 2008;105(48):18919-18923.

70. Tan X, et al. Beta-catenin deletion in hepatoblasts disrupts hepatic morphogenesis and survival during mouse development. Hepatology. 2008;47(5):1667-1679.

71. Apte $U$, et al. beta-Catenin is critical for early postnatal liver growth. Am J Physiol Gastrointest Liver Physiol. 2007;292(6):G1578-G1585.

72. Ishikawa T, et al. Hepatocyte growth factor/cmet signaling is required for stem-cell-mediated liver regeneration in mice. Hepatology. 2012; 55(4):1215-1226.

73. Van Hul NK, barca-Quinones J, Sempoux C, Horsmans Y, Leclercq IA. Relation between liver progenitor cell expansion and extracellular matrix deposition in a CDE-induced murine model of chronic liver injury. Hepatology. 2009;49(5):1625-1635.

74. Lorenzini S, et al. Characterisation of a stereotypical cellular and extracellular adult liver progenitor cell niche in rodents and diseased human liver. Gut. 2010;59(5):645-654.

75. Spee B, et al. Characterisation of the liver progenitor cell niche in liver diseases: potential involvement of Wnt and Notch signalling. Gut. 2010;59(2):247-257.

76. Lin SL, et al. Macrophage Wnt7b is critical for kidney repair and regeneration. Proc Natl Acad Sci US A. 2010;107(9):4194-4199.

77. Reddy SM, et al. Phagocytosis of apoptotic cells by macrophages induces novel signaling events leading to cytokine-independent survival and inhibition of proliferation: activation of Akt and inhibition of extracellular signal-regulated kinases 1 and 2 . J Immunol. 2002;169(2):702-713.

78. Van HN, Lanthier N, Espanol SR, Abarca QJ, Van RN, Leclercq I. Kupffer cells influence parenchymal invasion and phenotypic orientation, but not the proliferation, of liver progenitor cells in a murine model of liver injury. Am J Pathol. 2011;179(4):1839-1850.

79. Clouston AD, Powell EE, Walsh MJ, Richardson MM, Demetris AJ, Jonsson JR. Fibrosis correlates with a ductular reaction in hepatitis C: roles of impaired replication, progenitor cells and steatosis. Hepatology. 2005;41(4):809-818.

80. Gouw AS, Clouston AD, Theise ND. Ductular reactions in human liver: Diversity at the interface. Hepatology. 2011;54(5):1853-1863.

81. Sekiya S, Suzuki A. Intrahepatic cholangiocarcinoma can arise from Notch-mediated conversion of hepatocytes. J Clin Invest. 2012;122(11):3914-3918.

82. Fan B, et al. Cholangiocarcinomas can originate from hepatocytes in mice. J Clin Invest. 2012;122(8):2911-2915.

83. Wei W, Chua MS, Grepper S, So S. Small molecule antagonists of Tcf4/beta-catenin complex inhibit the growth of HCC cells in vitro and in vivo. Int J Cancer. 2010;126(10):2426-2436. 